\title{
Resiliência no trauma - a possibilidade de manejo na Terapia Cogni- tivo-Comportamental
}

\author{
Resilience in trauma - the possibility of management in Cognitive-Behavioral Therapy
}

Laísa Silva Kasmalski de Moraes*, Fátima Niemeyer da Rocha

\begin{abstract}
Resumo
Como citar esse artigo. de Moraes, L.S.K. \& da Rocha, F.N. Resiliência no trauma - a possibilidade de manejo na Terapia CognitivoComportamental. Revista Mosaico. 2017 Jan./Jun.; $07 \quad$ (2): 03-10.

O presente artigo aborda os principais conceitos de resiliência e sua construção do ponto de vista psicológico, com o objetivo de investigar o desenvolvimento da resiliência diante do trauma psicológico. Apresenta uma breve discussão sobre o manejo psicoterápico a partir da Terapia Cognitivo-Comportamental (TCC), tendo em vista a possibilidade de o psicólogo auxiliar na racionalização e na organização das cognições do paciente para que este possa alcançar funções adaptativas, na busca do desenvolvimento de um processo resiliente. Além disso, a articulação entre métodos e/ou técnicas da TCC e os fatores preponderantes da resiliência, envolve também as principais estratégias de enfrentamento das situações adversas e/ou traumáticas, como, por exemplo, o coping. Assim, o trabalho tem como foco central a resiliência psicológica individual enquanto fenômeno processual, que pode ser construída no trabalho psicoterápico, levando em conta fatores biológicos, sociais, psicológicos e comportamentais.

Palavras-Chave: Resiliência; Trauma; Terapia Cognitivo Comportamental.
\end{abstract}

\begin{abstract}
This article addresses the main concepts of resilience and its construction from a psychological point of view aiming to study the development of resilience in face of psychological trauma. It presents a brief discussion about the psychotherapeutic management from the Cognitive-Behavioral Therapy (CBT), considering the possibility of a psychologist assist in the rationalization and organization of the patient's cognitions so the patient, can achieve adaptive functions in order to get development of a resilient process. In addition, the articulation between CBT methods, techniques and the prevailing factors of resilience, also involve the main confrontation strategies of adverse and traumatic situations, such as coping. Thus, this article has as its central focus the psychological resilience of an individual as a procedural phenomenon, which can be constructed in the psychotherapeutic work, taking into account biological, social, psychological and behavioral factors.

Keywords: Resilience; Trauma; Cognitive Behavioral Therapy.
\end{abstract}

\section{Introdução}

Diante de situações adversas, alguns sujeitos desenvolvem psicopatologias, enquanto outros demonstram-se resilientes. Muitos estudos têm destacado o caráter processual da resiliência como importante para o manejo de situações ou eventos traumáticos, o que sugere a possibilidade de se trabalhar com os princípios da resiliência para auxiliar terapeuticamente indivíduos a desenvolverem comportamentos adaptativos frente as adversidades.

O conceito de resiliência envolve processos que explicam a "superação" de crises e adversidades em indivíduos, grupos e organizações, sendo fundamental no processo de intervenção terapêutica. Quando o terapeuta consegue, junto com o paciente, identificar comportamentos que facilitem o desenvolvimento da resiliência, este elabora formas de manejo das situações de maneira mais adaptativa.

Determinados padrões comportamentais podem denunciar a existência de comportamentos disfuncionais, decorrentes de traumas/situações adversas. No entanto, os eventos traumáticos, por si só, não são determinantes isolados ou exclusivos do

Afiliação dos autores: † USS - Universidade Severino Sombra, Vassouras-RJ. 
desenvolvimento de transtornos psicológicos, porque mesmo as experiências potencialmente intensas e devastadoras provocam efeitos variáveis nas pessoas.

O objetivo deste trabalho foi abordar o constructo resiliência no contexto da psicoterapia e a sua aplicabilidade, destacando as variáveis subjetivas e o contexto histórico-social que influenciam no seu desenvolvimento. Foi desenvolvido sob a perspectiva da TCC, que utiliza o conceito de resiliência e que possui uma gama de técnicas diretivas comprovadamente eficazes no espaço clínico em paciente que sofreram trauma. Buscou-se compreender porque alguns indivíduos já demonstram, naturalmente, fatores resilientes, investigando-se a possibilidade de despertar esses fatores em outros sujeitos. Também procurou-se identificar estratégias de enfretamento dos indivíduos diante de situações de vulnerabilidade e apresentar as principais variáveis constituintes do comportamento resiliente e sua aplicabilidade clínica.

Partiu-se da premissa, desenvolvida por Beck (2013), de que, no decorrer da vida, as pessoas constroem crenças sobre suas habilidades para enfrentar os problemas e dificuldades. Tais crenças e valores formam a estrutura da pessoa, como matrizes que organizam as suas ideias mais básicas. No âmbito da Psicologia Cognitiva recebem a denominação de Esquemas Básicos de Crenças. Assim, a partir da Teoria Cognitivo-Comportamental, descrevemos uma proposta de intervenção para que o paciente desenvolva comportamentos resilientes para lidar com as circunstâncias traumáticas, e desenvolver seu autoconhecimento.

\section{O conceito de Resiliência}

Ao observarmos o comportamento humano percebemos que alguns sujeitos demonstram mais vulnerabilidade diante de situações de estresse, enquanto outros apresentam uma incrível capacidade de adaptação. Quais seriam as variáveis que viabilizam a alguns a dita "superação" de situações adversas, de modo que, posteriormente, não provoquem interferência no seu desenvolvimento emocional?

Por se tratar de um conceito ainda em construção, os teóricos da resiliência apresentam diferentes definições para o termo; mas, até certo ponto, podese dizer que essas definições são complementares. Para citar alguns exemplos: segundo Placco (2001), resiliência compreende a capacidade de, frente às adversidades, reagir de forma consistente, flexível, positiva e perseverante; buscar estratégias para preservar-se psicologicamente e recuperar-se; manter um equilíbrio dinâmico durante e após os embates; não se deixar consumir pelas demandas adversas; e superar-se e superar as pressões de seu mundo com um autoconceito realista, autoconfiança e senso de autoproteção; Grotberg (2006 citado por SECUNHO, 2012 , p. 19.) define resiliência como "a capacidade humana para enfrentar, sobrepor-se e sair fortalecido ou transformado por experiências de adversidade"; de acordo com Moraes e Rabinovich (1996 citado por BARREIRA e NAKAMURA, 2006, p. 78) resiliência é "uma combinação de fatores que auxiliam os indivíduos a enfrentarem e superarem problemas e adversidades na vida"; e Flach (1991 citado por PINHEIRO, 2004, p. 69) usa o termo para se referir às forças psicológicas e biológicas requeridas para o indivíduo ser bem-sucedido diante das mudanças na vida; ele denomina de resiliente a pessoa que demonstra habilidade para "reconhecer a dor, perceber seu sentido e tolerá-la até resolver os conflitos de forma construtiva".

Ao longo do seu desenvolvimento, todos os indivíduos estarão sempre sujeitos a enfrentar situações adversas. Porém, alguns conseguem superar-se, vivenciando as adversidades de forma construtiva, desenvolvendo recursos de enfrentamento e transformando a vulnerabilidade em potencialidade e amadurecimento, enquanto outros se tornam mais vulneráveis e sucumbem frente às circunstâncias adversas. (ASSIS et al., 2006)

Assim, as diversas perspectivas do processo resiliente, apesar de parecerem dicotômicas, podem ser vistas como complementares para a construção do constructo resiliência.

E não há como falar de resiliência sem relacionála ao conceito de coping, visto que os dois conceitos estão intimamente relacionados. Segundo Antoniazzi et al. (1998, p. 273) o coping pode ser descrito como "o conjunto das estratégias utilizadas pelas pessoas para adaptarem-se a circunstâncias adversas ou estressantes". Lazarus e Folkman (1984 citados por ANTONIAZZI et al., 1998, p. 276) apresentam o coping como "um conjunto de esforços, cognitivos e comportamentais, utilizado pelos indivíduos com o objetivo de lidar com demandas específicas, internas ou externas, que surgem em situações de stress e são avaliadas como sobrecarregando ou excedendo seus recursos pessoais". E Yunes e Szymanski (2001 citados por SACHUK e CANGUSSU, 2008 , p. 4) destacam que o conceito de coping vem frequentemente acompanhado de palavras como habilidades, estratégias, comportamentos, estilos, respostas ou recursos.

Então, na relação entre resiliência e coping, a resiliência diz respeito às pessoas que conseguem se adaptar e superar as adversidades, e o coping enfoca as estratégias que as pessoas utilizam para enfrentar as adversidades. Embora ambos os conceitos estejam ligados a situações de alto estresse, quando falamos de coping, de uma forma geral, nos referimos a 
estratégias de enfrentamento, aquém do resultado alcançado, enquanto a resiliência foca, justamente, nos resultados a partir das estratégias utilizadas, o que, na verdade, estaria se referindo a uma adaptação eficaz diante de adversidades.

É necessário, ainda, considerar a resiliência a partir de três polos temáticos principais, propostos por Deslandes e Junqueira (2003citados por TABOADA et al., 2006, p. 105):

1) Resiliência enquanto processo de adaptação x superação - Ralha-Simões (2001 citado por PINHEIRO, 2004, p.70) destaca que a resiliência se refere a possibilidade de flexibilidade interna da pessoa, que the torna possível interagir com êxito, modificando-se de forma adaptativa em face dos confrontos adversos com o meio exterior. Luthar (2000 citado por INFANTE, 2005, p. 33), trata resiliência como "um processo dinâmico que tem como resultado a adaptação positiva em contextos de grande adversidade". E Walsh (2005 citado por ZAPPE et al, 2015, p. 222) versa sobre o termo "adaptação flexível", o qual remete tanto a presença de modificação, quanto de crescimento.

Em contrapartida, alguns autores defendem a ideia de resiliência como processo de superação, como Placco (2001), que a compreende como a capacidade que o indivíduo tem de responder de forma mais consistente aos desafios e dificuldades, de reagir com flexibilidade e capacidade de recuperação diante desses desafios e circunstâncias desfavoráveis, apresentando uma atitude otimista, positiva e perseverante, mantendo um equilíbrio dinâmico no decorrer e após a adversidade. Para ele, a resiliência pode ser concebida como uma característica da personalidade que, ativada e desenvolvida, possibilita ao sujeito superar as pressões de seu meio, desenvolver um autoconceito realista, autoconfiança e um senso de autoproteção que não desconsidera a abertura ao novo, às mudanças e ao outro. E Assis et al. (2006) e Walsh (2003 citado por BRANDÃO et al., 2011, p. 266) concebem a resiliência como uma oportunidade de recuperação, que supera o fato da pessoa se recuperar de um dano, pois demanda superação do que se era, além de crescimento pessoal.

Questiona-se, com relação a resiliência, se é uma capacidade que o indivíduo possui de se manter saudável apesar das adversidades ou se implica em progresso e desenvolvimento pessoal pelo enfrentamento das adversidades. Segundo Deslandes e Junqueira (2003 citados por TABOADA et al., 2006, p. 107), a resiliência considerada "apesar da adversidade" remete ao esforço da pessoa manter as características que já possuía antes da situação desagradável ou voltar ao estado de equilíbrio anterior. Essa capacidade resultaria de características pessoais, assim como dos vínculos de afeto e confiança estabelecidos com o meio no qual convive, conseguindo aprender algo frente ao problema e desenvolvendo comportamentos de adaptação ao que é esperado pela sociedade.

Por outro lado, na resiliência considerada "através da adversidade" o indivíduo se caracteriza por um rol de habilidades e competências tanto resultantes do processo resiliente, quanto por propiciadores do próprio processo. Os fatores de risco são vistos como oportunidades de superação dos próprios limites. Neste sentido, a resiliência "traz consigo a possibilidade de a experiência traumática ser elaborada simbolicamente, para que, futuramente, sirva como subsídio para novas situações estressantes". (DESLANDES e JUNQUEIRA, 2003 citado por TABOADA et al., 2006, p. 107) Na ideia de superação há a possibilidade de construção, aprendizagem ou desenvolvimento de características ditas resilientes. 2) Resiliência enquanto um fator inato $x$ fator adquirido - A resiliência é abordada ora com ênfase no indivíduo ora no meio. De acordo com Taboada et al. (2006, p. 108), algumas pesquisas focam as capacidades e habilidades da pessoa de modo descontextualizado, considerando a resiliência como um traço ou característica de personalidade e identificando fatores inatos no processo resiliente. Outras pesquisas, que enfocam o meio social, argumentam que a resiliência pode ser vista como uma habilidade social, podendo (e devendo) ser aprendida e estimulada. Este autor também afirma que "o meio tornar-se-ia então a chave principal de um processo que possui fortes indícios de possuir componentes inatos." (p. 109) 3) Resiliência como algo circunstancial $\mathrm{x}$ fator permanente - Taboada et al. (2006) argumenta que os termos "crianças resilientes" e "tornar os jovens resilientes" apontam para a ideia de que uma pessoa que não era resiliente pode tornar-se. Assim, um indivíduo resiliente é identificado através das habilidades e competências consideradas típicas da resiliência, e espera-se que ele atue conforme as habilidades apresentadas diante das adversidades, sejam elas quais forem.

Ralha-Simões (2001 citado por PINHEIRO, 2004, p. 70) a define como uma especificidade estrutural do desenvolvimento psicológico, que se traduz na capacidade que determinadas pessoas, grupos ou instituições possuem para evitar, enfrentar ou mesmo ultrapassar os efeitos desestruturantes da exposição a certas experiências. Estaria, assim, implícita a noção de resiliência como uma facilitação da interação eficaz com o meio, adquirida através de uma perpétua modificação estrutural interna.

Por outro lado, a concepção de resiliência como algo circunstancial aparece em Silva et al. (2005), referindo-se à capacidade dos seres humanos de enfrentar e responder de forma positiva às experiências que possuem elevado potencial de risco para a saúde e desenvolvimento do indivíduo. Aponta 
para um fenômeno atrelado à interdependência entre os múltiplos contextos com os quais o sujeito interage, cuja presença é observada quando este passa por uma situação adversa, seja esta de caráter temporário ou constante em sua vida.

A maioria dos estudos expressa que a resiliência não é estável, como Rutter (2006 citado por TABOADA et al., 2006), que concebe a resiliência diretamente ligada a processos dinâmicos, ao invés de fatores estáticos.

\section{A resiliência frente ao trauma}

Para identificar um indivíduo como resiliente é necessário que tenha estado frente a uma ameaça significativa, como uma exposição a um estado de alto-risco ou adversidade grave ou evento traumático; segundo, que a qualidade da adaptação seja boa. Indivíduos não são considerados resilientes se nunca tiverem sofrido uma ameaça significativa. (MASTEN e COATSWORTH, 1998; MASTEN, 2001 citados por VILETE, 2009). Na perspectiva do trauma, a resiliência é uma adaptação eficaz, apesar da presença de ameaças significativas a integridade pessoal e física. (AGAIBI e WILSON, 2005 citados por VILETE, 2009) Para Bonanno (2004 citado VILETE, 2009) essa seria a resposta mais comum a um trauma potencial, conclusão que parece baseada na menor proporção de um Transtorno do Estresse Pós-Traumático (TEPT).

De acordo com Peres et al. (2005, p. 131), os eventos traumáticos em si não são determinantes isolados ou exclusivos do desenvolvimento de transtornos psicológicos, pois as experiências potencialmente intensas e devastadoras possuem efeitos variáveis.

Um trauma ocorre quando as defesas psicológicas são transgredidas. (PERES et al., 2005, p. 133) Segundo Costa (2007), o trauma psíquico pode ser considerado como uma espécie de ferida, uma cisão na "pele" psíquica. Esta ferida ocorre quando os mecanismos de defesa não conseguem, devido ao impacto provocado pela carga emocional excessiva do trauma, assegurar o equilíbrio, deixando uma cisão aberta.

A expressão "pele psíquica" leva a pensar na resiliência como a sua flexibilidade para resistir ao impacto ou abalo pela acentuada carga emocional do trauma, de forma que essa pele não se rompa.

O CID-10 (2008) e o DSM-5 (2014) apresentam diferenças marcantes nos critérios para definição de TEPT. A CID-10 (2008), na descrição do "Estado de "stress' pós-traumático" define evento traumático como uma situação ou evento estressante (de curta ou longa duração), de natureza excepcionalmente ameaçadora ou catastrófica, e que provocaria sintomas evidentes de perturbação na maioria dos indivíduos. Já no DSM-5 (2014), ao descrever o "Transtorno de estresse pós-traumático" apresenta o evento traumático de forma mais abrangente, incluindo qualquer evento que represente uma ameaça a própria integridade física ou de outras pessoas. Os eventos traumáticos são definidos, não apenas em função das características objetivas da experiência vivida, mas também em função da reposta subjetiva de intenso medo, impotência ou horror diante do evento.

Para Hoge et al. (2007 citado por VILETE, 2009), diante de um evento traumático o indivíduo resiliente seria aquele que experimenta um trauma e não desenvolve TEPT. Bonanno (2006 citado por VILETE, 2009) também considera a resiliência como ausência de sintomas de TEPT ou a presença de apenas um sintoma desse transtorno.

A forma como as pessoas processam e/ou manejam o acontecimento estressante é um dos fatores determinantes para que o trauma se configure. Segundo Peres et al. (2005, p. 133), a caracterização de um evento como traumático não depende somente do estimulo estressor, mas da tendência de processamento perceptual do indivíduo. A percepção de si mesmo e os diálogos internos após a ocorrência do evento traumático são preditores de resultados psicológicos satisfatórios ou não. Os diálogos internos de autopiedade, desamparo, auto vitimização e auto depreciação podem realçar as emoções negativas relacionadas a memória traumática e exacerbar o sofrimento psicológico. As pessoas que cultivam diálogos internos de enfrentamento, procurando modificar o presente positivamente, superam com maior facilidade traumas psicológicos.

Essa variabilidade de concepções viabiliza a hipótese da construção de fatores para a resiliência em psicoterapia.

\section{Resiliência e terapia cognitivo- comportamental - uma proposta de manejo}

Focalizando o processo de construção da resiliência individual, investigou-se uma estrutura de trabalho psicoterápico, embasado na TCC, para o seu fortalecimento ou estruturação a partir das técnicas utilizadas pelo psicólogo no espaço clínico.

Consoante a Gomes (2010), a resiliência é vinculada a fatores de risco e de proteção. Os fatores de risco envolvem os fatos traumáticos ou estressantes e que deixam marcas; e os fatores de proteção são os apoios que protegem o indivíduo desses impactos traumáticos, como autoestima positiva, humor, otimismo, temperamento maleável, disciplina pessoal, 
estabilidade emocional, capacidade de adaptação, talento e competência, entre outros.

Tendo em vista que do equilíbrio ou (re) equilíbrio entre os fatores de risco e de proteção surge a resiliência e que os traumas ou impactos são aliviados por esses fatores que, de alguma forma, "abraçam" e protegem a pessoa, é importante identificar a possiblidade de construção de tais fatores dentro da psicoterapia, tendo em vista um desenvolvimento resiliente.

A finalidade das psicoterapias aplicadas às vítimas de traumas psicológicos é atribuir, gradualmente, novos significados emocionais à experiência traumática passada, que não ocorre mais no presente. (PERES ET AL., 2005) Nesse sentido, mais importante do que a situação real, é a avaliação que o indivíduo faz a respeito dela.

Sarma (2008 citado por BARBOSA, 2011) argumenta que as pessoas possuem áreas que elas mobilizam em situações que envolvem adversidades, desafios e problemas, que demandam a necessidade de superação. Essas áreas estimulam a manifestação de crenças profundas, relativas, por exemplo, a capacidade de resolução de problemas, de se sentir amado, de se perceber como uma pessoa interessante para as outras, de administrar suas emoções e de lidar com as alterações fisiológicas, entre outras.

Falcone (2001 citado por DEL PRETTE e DELL PRETTE, 2006) explica que a dinâmica da TCC aponta para a mudança das crenças disfuncionais que produzirá modificação nas emoções e nos comportamentos desadaptados. "Suas demandas incluem: ênfase no aqui-e-agora; sessões estruturadas e contínuas; solução de problemas; reestruturação de pensamentos disfuncionais; papel ativo por parte do terapeuta e do paciente; definição de metas; adesão as tarefas de autoajuda" (p. 149). Como relatado por Norte et al. (2011), através da TCC pode-se promover melhoras nas cognições e no comportamento relacionados aos sintomas do TEPT, em que "cognições de vulnerabilidade" são substituídas por "cognições adaptativas e realistas", de acordo com as situações apresentadas.

Um funcionamento resiliente parece surgir de interações de fatores herdados, características individuais e fatores experienciais através do tempo (circunstâncias de vida, familiares e sociais), numa conjunção de fatores genético/biológicos, cognitivos e interpessoais. (COLLISHAW, 2007 citado por VILETE, 2009) Entretanto, essas características não devem dar a ilusória ideia de resistência absoluta diante do estresse, pois, segundo Rutter (1993 citado por BARREIRA E NAKAMURA, 2006), a resistência ao estresse é relativa e suas bases são tanto constitucionais quanto ambientais, sem uma base fixa e que variam de acordo com as circunstâncias.
É importante lembrar que a resiliência não envolve a ausência de se experimentar um afeto negativo, mas a habilidade de se recuperar mais rápida e facilmente e retornar a um estado de afeto positivo, além de uma aumentada capacidade de aprender com a experiência de afeto negativo. (CURTIS e CICHETTI, 2003 citado por VILETE, 2009)

A maioria das técnicas comportamentais, usadas na TCC, destinam-se a "ajudar as pessoas a: 1) romper padrões de evitação ou desesperança; 2) enfrentar gradativamente situações temidas; 3) desenvolver habilidades de enfrentamento; e 4) reduzir emoções dolorosas ou excitação". (WRIGHT et al., 2008, p. 29)

Também é importante auxiliar o paciente no desenvolvimento da autoestima; sobre isso Deffenbacher (2002) propõe auxiliar o paciente incentivando suas tentativas de enfrentamento, focando no processo e não no resultado a priori. As tentativas de enfrentamento devem ser incentivadas, inclusive quando o resultado não é positivo.

Da mesma forma, é útil uma diferenciação entre processo e resultado. Se o paciente tenta (processo), pode sentir-se bem, inclusive se o resultado é negativo, vendo-o como oportunidade para reciclar a solução do problema. Se não tenta, está quase assegurada uma avaliação pobre do processo e do resultado. (DEFFENBACHER, 2002) Por exemplo, um paciente poderia pensar: "me aborreci mais do que queria, mas mantive bem o controle"; um homem socialmente ansioso: "Bom, não consegui um encontro, mas controlei minha ansiedade e me atrevi a pedir. Sinto-me bem por isso. Talvez na próxima vez tenha mais sorte." Estes pensamentos mantêm o paciente envolvido na solução do problema e evitam uma frustação desnecessária.

Despertar esse olhar sobre o processo é um vislumbre promissor no trabalho clínico de auxílio ao paciente para desenvolver autoestima e um olhar positivo quanto as suas circunstâncias e possibilidades.

Com o objetivo de auxiliar o paciente, que se encontra numa situação pós-trauma, a reconhecer e a modificar o pensamento desadaptativo, para alcançar emoções positivas, destaca-se o 'questionamento socrático' como uma técnica útil para que o paciente possa se situar. Segundo Wright et al. (2008, p. 28), o questionamento socrático consiste em fazer perguntas que estimulem o paciente e despertem a curiosidade e o desejo de inquirir.

Uma forma especial de questionamento socrático é a "descoberta guiada", por meio da qual o terapeuta faz uma série de perguntas indutivas para revelar padrões disfuncionais de pensamento ou comportamento, tais como: 


\begin{abstract}
“ Quais são as evidências de que o seu pensamento é verdadeiro? Quais são as evidências em contrário? Qual seria uma forma alternativa de encarar esta situação? O que de pior poderia acontecer e como você lidaria com isso? $\mathrm{O}$ que de melhor poderia acontecer? Qual é o resultado mais realista desta situação? Qual é o efeito de acreditar no seu pensamento automático e qual seria o efeito de mudá-lo? Se o [seu amigo ou familiar] estivesse nesta situação e tivesse o mesmo pensamento automático, que conselho você lhe daria? O que devemos fazer?" (BECK, 2013, p. 43)
\end{abstract}

Na sessão de terapia, durante a discussão de um problema, o terapeuta evoca cognições do paciente (pensamentos automáticos, imagens e/ou crenças), procura averiguar qual cognição ou cognições são mais perturbadoras, faz uma série de perguntas para ajudá-lo a olhar para o problema com distanciamento (isto é, encarar as suas cognições como ideias, não necessariamente como verdades) e a avaliar a validade e a utilidade das cognições e/ou "descatastrofizar" seus medos. A pessoa ativa processos cognitivos para identificar a situação crítica com a qual se depara; checar suas condições atuais, experiências eficazes e ineficazes anteriores, nível e grau de risco envolvido nesta; eanalisar os recursos disponíveis e as alternativas possíveis para lidar com o problema. (FOLKMAN E LAZARUS, 1980 citados por LISBOA et al., 2002, p.346)

O treinamento em habilidades sociais (THS), por sua vez, pode ser definido como "um enfoque geral da terapia, dirigido a incrementar a competência da atuação em situações críticas da vida". (GOLDSMITH e MCFALL, 1975 citado por CABALLO, 2002, p. 367). Segundo Caballo (2002) o processo do THS implicaria, em seu desenvolvimento completo, quatro elementos de forma estruturada:

- Treinamento em habilidades - onde se ensina comportamentos específicos, que são praticados e integrados ao repertório comportamental do sujeito. - Redução da ansiedade em situações problemáticas - esta pode ser alcançada de forma indireta, pela adoção de um novo comportamento mais adaptativo que supostamente é incompatível com a resposta da ansiedade (WOLPE, 1958 citado por CABALLO, 2002, p. 367). Se o nível de ansiedade é muito elevado pode-se empregar diretamente uma técnica de relaxamento.

- Reestruturação Cognitiva - na qual se pretende modificar crenças, cognições e/ou atitudes do sujeito buscando a aquisição de novos comportamentos. $\mathrm{O}$ primeiro passo consiste em aumentar a percepção do paciente sobre os processos de pensamento mais frequentes, como pensamentos automáticos dos quais o paciente não tem conhecimento (BECK, 1976 citado por DEFFENBACHER, 2002, p. 569); a seguir, recolhe-se evidências prós e contras essas cognições. Muitos pacientes começam a corrigir os pensamentos disfuncionais à medida que vão aparecendo.
- Treinamento em solução de problemas - onde se ensina o sujeito a perceber corretamente os "valores" de todos os parâmetros situacionais relevantes, a processar os "valores" destes parâmetros para gerar respostas potenciais, a selecionar uma dessas respostas e usá-la de uma forma que maximize a probabilidade de alcançar o objetivo que impulsionou a comunicação interpessoal.

Todavia, antes de ensinar habilidades sociais ao paciente, o terapeuta deve avaliar o nível das habilidades que ele já apresenta. Muitos sabem precisamente o que fazer e dizer em cada situação, mas têm dificuldade em usar esse conhecimento devido a pressupostos disfuncionais (por exemplo, "se eu expressar uma opinião eu vou ser ignorado"). Alguns pacientes apresentam habilidades sociais frágeis, de modo geral, ou são melhores em um estilo de comunicação, mas tem habilidade para adaptar o seu estilo. (BECK, 2013, p. 288-289)

Autores como Beck (2013) e Caballo (2002), entre outros, citam o uso da técnica do role-play ou dramatização, a qual pode ser usada para uma vasta variedade de propósitos, dentre eles na aprendizagem e na prática de habilidade sociais. Conforme Beck (2013), pode-se utilizar a dramatização para auxiliar o paciente a identificar seus pensamentos automáticos enquanto está sendo assertivo ou a prever pensamentos e sentimentos da outra pessoa quando os papeis forem trocados.

Embora haja uma gama de técnicas disponíveis, ressalta-se que o terapeuta cognitivo-comportamental não trabalha com fórmulas prontas. Essas técnicas são utilizadas de acordo com a necessidade de cada paciente, diante do qual devem ser muito bem averiguadas pelo terapeuta. Este deve ter a perspicácia profissional de flexibilizar o uso das técnicas, tendo por objetivo uma aplicação integrada de acordo com a singularidade de cada sujeito.

Além disso, é importante ter em vista que a tríade mente-corpo-meio deve ser avaliada em conjunto pelo terapeuta e que, a partir dessa visão, ele deve lançar mão do que melhor favorece a eficácia do trabalho terapêutico. Como citado por Balhs e Navolar (2004) o terapeuta deve utilizar o "conceito da estrutura 'biopsicossocial' na determinação e na compreensão dos fenômenos relativos a psicologia humana".

Do ponto de vista clínico, a abordagem do funcionamento da resiliência é complexa, porque aparece associada a múltiplos parâmetros, para onde convergem um conjunto de variáveis. Assim, a resiliência pode ser entendida como o próprio processo de transformação psíquica e o resultado deste trabalho mental, em termos da adaptabilidade e da interação com o meio psicoafetivo. (COSTA, 2007)

Observa-se, portanto, a possibilidade de se estabelecer um diálogo entre algumas técnicas da TCC e os fatores ligados a resiliência. Assim, consideramos 
promissor o trabalho psicoterápico embasado na TCC para o fortalecimento ou a estruturação do processo de construção da resiliência individual diante do trauma, a partir do manejo das técnicas utilizadas pelo psicólogo no espaço clínico.

\section{Considerações finais}

Mesmo diante da diversidade de conceitos atribuídos ao termo resiliência por diferentes estudiosos, pode-se considera-la como uma força intrínseca a todo ser humano, que reúne seus recursos biológicos, psíquicos e sociais na construção da sua capacidade de superação diante das situações adversas que ameaçam sua existência. Ou seja, envolve a capacidade do ser humano de construir uma trajetória positiva de vida e de desenvolvimento, apesar das condições adversas e traumáticas que o cercam.

Perceber que a resiliência se constitui não somente a partir das caraterísticas pessoais do sujeito, mas também das suas relações no contexto sociocultural em que se insere, possibilita a compreensão de que, na simultaneidade entre o individual e o social, ao mudar suas interpretações e ações, muda-se o contexto em que ele vive.

Nesse sentido, a ideia de se trabalhar a resiliência psicológica para enfrentamento da situação traumática, com foco psicoterápico no indivíduo, é adequada à visão biopsicossocial da TCC, segundo a qual a articulação entre as ideias e as técnicas, associadas aos fatores ligados a resiliência, podem produzir resultados clínicos eficazes. E a resiliência deve ser reconhecida como um importante conjunto de processos que possibilita ajustes cognitivos, emocionais e relacionais/sociais para obter saúde e bem-estar.

Ao apontar para o caráter processual da resiliência, almeja-se que, através do trabalho psicoterápico ativo entre terapeuta e paciente, possam ser construídos "caminhos" resilientes. É importante salientar que todo sujeito tem um potencial de superação, adaptação e crescimento, e os meios para o crescimento devem ser buscados sempre.

Cabe ao psicólogo ter em mente que, apesar dos danos e prejuízos causados pelo trauma, podese criar recursos adaptativos e, assim, o sujeito pode passar a desenvolver um processo resiliente. Afinal, não existe resiliência sem adversidade ou, até mesmo, sem trauma; mas é, justamente, neste ponto que, por intermédio da resiliência, pode-se evitar o desenvolvimento de psicopatologias. Para isso, o terapeuta pode se utilizar de métodos da TCC para a construção de fatores resilientes junto ao paciente.

\section{REFERÊNCIAS}

ANTONIAZZI, A. S.; DELL' AGLIO, D. D.; BANDEIRA, D. R. O conceito de Coping: uma revisão teórica. Estudos de Psicologia, Universidade Federal do Rio Grande do Sul, v.3, n.2, p.273-294, 1998.

ASSIS, S. G.; PESCE, R. P; AVANCI, J. Q. Resiliência: enfatizando a proteção dos adolescentes. Porto Alegre: Artmed, 2006.

BAHLS, S. C.; NAVOLAR, A B. B. Terapias cognitivo comportamentais: conceitos e pressupostos teóricos. Revista Eletrônica Psicologia, Curitiba, n. 4, p 1-11, 2004 .

BARBOSA, G. A aplicação e interpretação do conceito de resiliência em nossa teoria. Anais do $\mathbf{1 1}^{\circ}$ congresso de stress da isma-br. Porto Alegre: 2011.

BARREIRA, D. D.; NAKAMURA, A. P. Resiliência e a autoeficácia percebida: articulação entre conceitos. Aletheia, Universidade Luterana do Rio Grande do Sul, n.23, p.75-80, 2006.

BECK, J. S. Teoria cognitivo-comportamental: Teoria e prática. Porto Alegre: Artmed, 2013

BRANDÃO, J. M; MAHFOUD, M.; NASCIMENTO, I. F. G. A construção do conceito de resiliência em psicologia: discutindo as origens. Paidéia, V.21, N.49, P 263- 271, 2011.

CABALLO, V. E. O treinamento em habilidades sociais.In CABALLO, V. E. (Org.). Manual de técnicas de terapia e modificação do comportamento. São Paulo: Santos, 2002. p.361-398.

CID-10. Classificação Estatística Internacional de Doenças e Problemas Relacionados à Saúde. São Paulo: Edusp, 2008.

COSTA, M. S. A resiliência: vitimização da Criança e do Adolescente. Dissertação (Mestrado em Medicina). Faculdade de Medicina de Lisboa. Lisboa, 2007.

DEFFENBACHER, J. L. S. A inoculação do estresse. In CABALLO, V. E. (Org.). Manual de técnicas de terapia e modificação do comportamento. São Paulo: Santos, 2002. p.557-579.

DEL PRETTE, Z. A. P.; DEL PRETTE, A. Estudos sobre habilidades sociais e relacionamento interpessoal. São Paulo: Casa do Psicólogo, 2006.

DSM-5. Manual Diagnostico e Estatístico de Transtornos Mentais. American Psychiatnc Association. Porto Alegre: Artmed, 2014.

GOMES, V. Jovens depois da chuva: Um estudo fenomenológico sobre resiliência. São Paulo: Clube de Autores, 2010.

INFANTE, F. A resiliência como processo: uma revisão da literatura recente. In MELILO, A.; OJEDA, E. N. S. e cols. (Orgs). Resiliência: descobrindo as próprias fortalezas. Porto Alegre: Artmed, 2005. p. 23-38.

LISBOA, C; KOLLER, S. H.; RIBAS, F. F.;BITENCOURT, K.; OLIVEIRA, L.; PORCIUNCULA, L. P. ; BUSNELLO, R. M. Estratégias de coping de crianças vítimas e não vítimas de violência doméstica. Psicologia: Reflexão e Crítica, Universidade Federal do Rio Grande do Sul, v. 15, n. 2, p. $345-$ $362,2002$.

NORTE, C. E.; SOUZA, G. G. L.; PEDROZO, A. L.; SOUZA, A. C. F. M.; FIGUEIRA, I.; VOLCHAN, E.; VENTURA, P. Impacto da terapia cognitivocomportamental nos fatores neurobiológicos relacionados à resiliência. Revista de Psiquiatria Clínica, Rio de janeiro, v.38, n.1, p. 43-45, 2011.

PERES, J. F. P; MERCANTE, J. P. P.; NASELlO, A. G. Promovendo Resiliência em Vítimas de Trauma Psicológicos. Revista Psiquiatria, Rio Grande do Sul, v.12, n.27, p 131-138, 2005.

PINHEIRO, D. P. N. A resiliência em discussão. Psicologia em Estudo, Maringá, v.9, n.1, p.67-75, jan/abril, 2004.

PLACCO, V. M. N. S. Resiliência e desenvolvimento pessoal. In TAVARES, J. (Org.) Resiliência e Educação. São Paulo: Cortez, 2001. Cap.04.

SACHUK, M. I.; CANGUSSU, E. T. Apontamentos iniciais sobre o conceito de resiliência. Serviço Social em Revista, Londrina, v. 11, n.01, p.2-14, jul/ dez. 2008. 
vida. Brasília: Theasurus, 2012.

SILVA, M. R. S.; LUNARDI, V. L.; LUNARDI, F. W. D.; TAVARES, K.

O. Resiliência e promoção da saúde. Texto e Contexto- Enfermagem,

Florianópolis, v.14(n. especial), p.95-102, 2005.

TABOADA, N. G; LEGAL, E. J; MACHADO, N. Resiliência: em busca de um conceito. Revista Brasileira Crescimento e Desenvolvimento

Humano, São Paulo, v.16, n.3, p.104-113, 2006.

VILETE, L. M. P. Resiliência a eventos traumáticos: conceito, operacionalização e estudo seccional. Tese de Doutorado em Saúde Pública. Escola Nacional de Saúde Pública Sérgio Arouca. Rio de Janeiro, 2009.

WRIGHT, J. H.; BASCO, M. R.; THASE, M. E. Aprendendo a Terapia Cognitivo- Comportamental: Um guia ilustrado. Porto Alegre: Artmed, 2008.

ZAPPE, G. J.; YUNES, M. A. M.; DELL'AGLIO, D. D. Psicologia dos desastres e resiliência comunitária: Reflexões sobre o incêndio da boate Kiss em Santa Maria. In COIMBRA, R. M.; MORAIS, N. A.; (Orgs.). A resiliência em questão: perspectivas teóricas pesquisa e intervenção. Porto Alegre: Artmed, 2015. p. 215-229. 